\title{
Degradação do Polipropileno durante a Extrusão e a Geração de Compostos Orgânicos Voláteis
}

\author{
Carlos A. Cáceres \\ Programa de Pós-Graduação em Ciência e Engenharia de Materiais, UFSCar \\ Sebastião V. Canevarolo \\ Departamento de Engenharia de Materiais, UFSCar
}

\begin{abstract}
Resumo: Estudou-se a degradação de um polipropileno copolímero não estabilizado durante a extrusão a diferentes temperaturas (de 180 a $240{ }^{\circ} \mathrm{C}$ ) e a conseqüente geração de compostos orgânicos voláteis (VOC). Os VOC foram coletados por meio de um condensador de gases, conectado na zona de desgaseificação da extrusora, e analisados por espectrometria no infravermelho (FTIR-ATR). A degradação do polímero foi acompanhada através da cromatografia de exclusão por tamanho e FTIR. À temperatura de $180^{\circ} \mathrm{C}$ a degradação do polipropileno não gerou quantidades coletáveis de compostos orgânicos voláteis. Por outro lado, a partir de $200{ }^{\circ} \mathrm{C}$ houve geração de compostos olefínicos, não voláteis à temperatura ambiente, que aumentaram em quantidade com o aumento da temperatura de extrusão. A Função de Distribuição de Cisão de Cadeia (CSDF) mostrou que a baixas temperaturas, 180 e $200{ }^{\circ} \mathrm{C}$, o mecanismo de degradação do polipropileno é preferencialmente por cisão de cadeia com alguma ramificação e/ou reticulação. Entretanto, nas temperaturas de extrusão mais altas, 220 e $240^{\circ} \mathrm{C}$, o mecanismo de degradação envolve exclusivamente cisão de cadeia e do tipo preferencial, ou seja, o número de cisões aumenta com o aumento da massa molar inicial da cadeia polimérica original.
\end{abstract}

Palavras-chave: Polipropileno, degradação termo-mecânica, distribuição de massa molar, cisão de cadeia, FTIR, CSDF.

\section{Polypropylene Degradation during Extrusion and the Formation of Volatile Organic Compounds}

Abstract: The thermo-mechanical degradation of an unstabilized polypropylene copolymer during extrusion at different temperatures (from 180 to $240{ }^{\circ} \mathrm{C}$ ) and the production of volatile organic compounds (VOC) were studied. VOC's were collected via a volatile condenser, fitted at the degassing exit of the extruder and analyzed via infrared spectroscopy (FTIR-ATR). The polymer degradation was followed via size exclusion chromatography and FTIR. At low extrusion temperatures $\left(180^{\circ} \mathrm{C}\right)$ the PP degradation produces undetectable quantities of VOC's. On the other hand, starting from $200{ }^{\circ} \mathrm{C}$, there is the formation of olefinic compounds of molecular weight not volatile at room temperature, which increase in quantity with the rise of the extrusion temperature. The Chain Scission Distribution Function (CSDF) shows that at low temperatures $\left(180\right.$ and $\left.200{ }^{\circ} \mathrm{C}\right)$ the $\mathrm{PP}$ degradation mechanism is preferentially via chain scission with some branching and/or crosslinking in the lower molecular weight. At higher extrusion temperatures $\left(220\right.$ and $\left.240{ }^{\circ} \mathrm{C}\right)$ the degradation mechanism is exclusively via chain scission and it is preferential, i.e., the number of chain scission increases with the increase in the initial molecular weight of the original polymeric chain.

Keywords: Polypropylene, thermo-mechanical degradation, molecular weight distribution, chain scission, FTIR, CSDF.

\section{Introdução}

O polipropileno isotáctico é um termoplástico semicristalino utilizado em varias aplicações de grande importância comercial. No entanto, o polipropileno homopolímero mostra algumas limitações como baixa tenacidade a baixas temperaturas. Modificações de rigidez e transparência no polipropileno podem ser realizadas, via copolimerização com outras olefinas como etileno ou buteno ${ }^{[1]}$. A versatilidade de processos de copolimerização tornou possível a produção de copolímeros e blendas, in situ, permitindo obterem-se materiais com diferentes conteúdos de comonômero. Os principais motivos para se fazer copolímeros aleatórios foram para melhorar as propriedades ópticas e diminuir a temperatura de fusão. Dependendo da reatividade do comonômero, condições de polimerização e sistema catalítico utilizado a distribuição das unidades do comonômero ao longo da cadeia pode variar muito, desde puramente aleatórios até a geração de longas sequiências ou blocos de um dos componentes ${ }^{[1]}$.

Hinsken et al. ${ }^{[2]}$ estudaram a degradação termo-oxidativa e termo-mecânica do polipropileno (PP) e polietileno de alta densidade (PEAD) sob múltiplas extrusões. No PP observaram um aumento de grupos carbonílicos e insaturações com o número de extrusões e uma redução da massa molar ponderal média. Os mecanismos de cisão das macro-

Autor para correspondência: Sebastião V. Canevarolo, Departamento de Engenharia de Materiais, UFSCar, Caixa Postal 676, CEP: 13560-905,

São Carlos, SP, Brasil. E-mail: caneva@ufscar.br 
moléculas do PP conduzem a uma redução da massa molar média que são causadas principalmente pela cisão- $\beta$, ruptura dos radicais peróxi e por cisalhamento. Por outro lado, o PEAD apresentou uma diminuição no MFI e um aumento na massa molar com o número de extrusões mostrando que o PEAD tem uma maior tendência à ramificação do que à cisão de cadeia.

Pinheiro et al. ${ }^{[3]}$ estudaram a degradação termo-mecânica do PEAD em uma extrusora dupla rosca sob várias condições de processamento. Análise de FTIR mostrou que existe um aumento na concentração de carbonilas e de grupos transvinilideno, enquanto que grupos vinil diminuem com o número de extrusões sendo que essa tendência se intensifica com o aumento da temperatura. O mecanismo de degradação termo-mecânica inicia-se com a cisão das cadeias mais longas, devido à sua maior probabilidade de emaranhamento. Por outro lado as cadeias menores, por apresentarem uma maior mobilidade não sofrem cisão, mas apresentam uma tendência à formação de ramificações.

Xiang et al. ${ }^{[4]}$ estudaram a emissão de compostos orgânicos voláteis (VOC) de um PP comercial sem estabilizantes, multiprocessado em uma injetora. Após cada ciclo de injeção o PP foi aquecido dinamicamente em um reator e os VOC foram monitorados através de um detector de ionização de chama. Os resultados mostraram que a quantidade máxima de VOC de cada ciclo não muda significativamente, enquanto os VOC cumulativos aumentam com o número de ciclos. Mudanças estruturais no polímero (analisadas com FTIR) em função do número de re-processamentos mostraram uma boa concordância com as mudanças reológicas acompanhadas por MFI.

De um ponto de vista ambientalista e em atendimento ao Clean Air Amendments de 1990 (CAAA90) indicou-se a redução de emissão de vários poluentes na atmosfera, e conseqüentemente as companhias foram forçadas a elaborar um inventário de suas emissões no meio ambiente. Em resposta a essa necessidade a Society of the Plastics Industry, Inc (SPI) nos USA organizou vários estudos para determinar os fatores de emissão durante a extrusão de alguns polímeros ${ }^{[5-7]}$. Adams et al. ${ }^{[7]}$ estudaram os fatores de emissão de compostos orgânicos voláteis e particulados gerados durante a extrusão a diferentes temperaturas e utilizaram vários tipos de PP comerciais homopolímeros e copolímeros. Os autores indicaram que os componentes gerados em maior quantidade foram particulados (compostos orgânicos voláteis de maior massa molar) e compostos orgânicos voláteis de menor massa molar como hidrocarbonetos $\left(\mathrm{C}_{2}-\mathrm{C}_{13}\right)$, etano, etileno, propileno. Em menor quantidade foram encontrados compostos oxigenados como aldeídos, cetonas e ácidos orgânicos.

A fração de cadeias clivadas $\left(f_{s}\right)$ durante a degradação de uma amostra dita original, não degradada, de referência ou polímero virgem, pode ser calculada quantificando-se a mudança na massa molar numérica média, pois essa é a única média que está diretamente relacionada com o número de moléculas ${ }^{[8]}$.

$$
f_{s}=\frac{\bar{M}_{n(0)}}{\bar{M}_{n(D)}}-1
$$

sendo $f_{\mathrm{s}}$ a fração de cadeias clivadas e $\overline{\mathrm{M}}_{\mathrm{n}}$ a massa molar numérica média com o índice (0) indicando antes e $(D)$ após a degradação.

Em trabalhos anteriores ${ }^{[9,10]}$ foi desenvolvida uma metodologia para entender melhor os processos de degradação termo-mecânica de poliolefinas, acompanhando-se o deslocamento de toda a curva de MWD das amostras degradadas com relação a uma curva de referência. Essa metodologia foi chamada de Função de Distribuição de Cisão de Cadeia (CSDF) e foi utilizada para acompanhar os processos de degradação termo-mecânica do polietileno de alta densidade ${ }^{[3]}$, polipropileno ${ }^{[9-11]}$ e poliestireno ${ }^{[8]}$ durante a extrusão.

Neste trabalho analisamos a degradação termo-mecânica de um polipropileno copolímero sem estabilizantes extrudado apenas uma vez, mas em diferentes temperaturas de extrusão. Compostos orgânicos voláteis gerados durante a extrusão foram coletados, analisados por FTIR-ATR e correlacionados com as mudanças estruturais do polímero processado.

\section{Experimental}

\section{Material}

Utilizou-se um polipropileno copolímero aleatório propeno - eteno na forma de pó nascente sem adição de nenhum tipo de estabilizante, fornecido pela BRASKEM S.A. O material foi processado logo depois de recebido.

\section{Extrusão}

O processamento foi realizado em uma extrusora dupla rosca modular, co-rotativa e totalmente interpenetrante, modelo ZSK-30 da marca Werner\&Pfleiderer. As condições operacionais foram as seguintes: velocidade de rotação da rosca de $70 \mathrm{rpm}$, taxa de alimentação de $2 \mathrm{~kg} / \mathrm{h}$ e perfil de rosca do tipo $2 \mathrm{~KB} 45^{[3]}$. A extrusão foi realizada mantendo-se constante todas as condições operacionais citadas anteriormente e variando unicamente a temperatura de processamento (180, 200, 220 e $240{ }^{\circ} \mathrm{C}$ ), sendo cada uma delas mantida fixa nas cinco zonas de aquecimento e no cabeçote da extrusora. A rotação de 70 rpm é a condição mínima de estabilidade desta extrusora para poder processar o material polimérico, aumentando o tempo de residência, permitindo intensificar o efeito da temperatura na degradação termomecânica durante a extrusão. Uma quantia suficiente de amostra foi coletada após cada extrusão para posterior análise. A coleta de voláteis foi feita através de um condensador de gases, do tipo "dedo frio" instalado na zona de desgaseificação da extrusora. Este acessório foi projetado e construído para nosso grupo de pesquisa (Figura 1). A cada processamento condensou-se voláteis no "dedo frio" por um tempo de 30 minutos de extrusão os quais foram posteriormente removidos via lavagem com $20 \mathrm{~mL}$ 


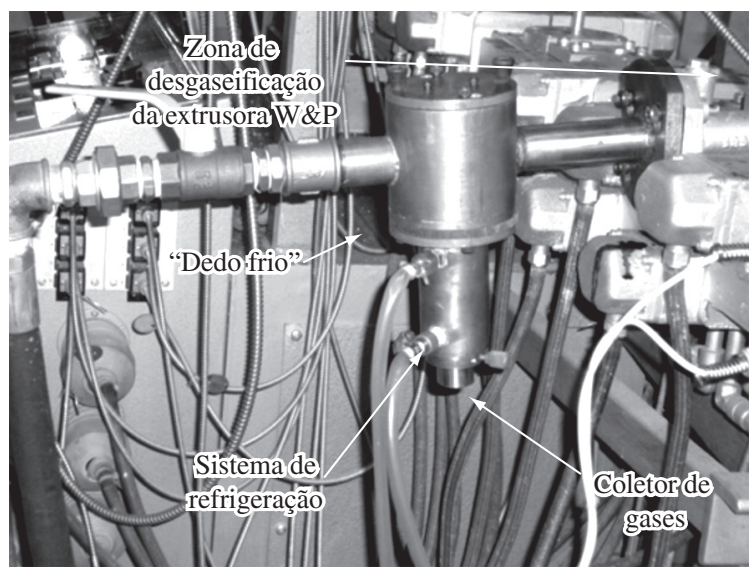

Figura 1. Foto do "dedo frio" acoplado na zona de desgaseificação da extrusora dupla rosca W\&P modelo ZSK-30.

de clorofórmio. Logo após a solução foi concentrada via evaporação na temperatura ambiente reduzindo-a até $5 \mathrm{~mL}$, para melhorar o nível de detecção dos VOC de maior massa molar. Devido à dificuldade de se processar o $\mathrm{PP}$ a $240{ }^{\circ} \mathrm{C}$ não foi possível, nesta temperatura, atingir o tempo mínimo necessário para coletar uma quantidade suficiente de VOC para análise.

\section{Cromatografia de exclusão por tamanho}

As curvas de distribuição de massa molar MWD foram obtidas em um cromatógrafo modelo $150 \mathrm{CV}$ (Waters), com detector por índice de refração e três colunas HT (Styragel). A curva de calibração universal foi realizada utilizando-se 14 padrões de poliestireno da Shodex Standard na faixa de $10^{3}-10^{6}$ Daltons. As amostras do PP virgem e as processadas a diferentes temperaturas de extrusão foram solubilizadas por uma hora à temperatura de $140{ }^{\circ} \mathrm{C}$ em $1,2,4$ Triclorobenzeno (grau HPLC), com antioxidante Irganox 1010. Após este procedimento cada solução foi filtrada e em seguida $200 \mu \mathrm{L}$ foi injetada no cromatógrafo SEC operando à temperatura de $135{ }^{\circ} \mathrm{C}$ e vazão de $1 \mathrm{~mL} / \mathrm{min}$.

\section{Função de distribuição de cisão de cadeia}

O cálculo da Função de Distribuição de Cisão de Cadeia (CSDF) foi realizado no programa CSDF $4.1^{[10]}$. Os valores da CSDF são calculados e apresentados como uma função da massa molar inicial, ou seja, da amostra de referência (polímero virgem), para maior informação desta metodologia ver as referências ${ }^{[9,10]}$. O mencionado programa, escrito em Excel, está disponível a todos os interessados, bastando apenas entrar em contato com os autores deste trabalho.

\section{Espectrometria no infravermelho}

Para a análise no infravermelho foram feitos filmes de $330 \mu \mathrm{m}$ de espessura via termo-prensagem, utilizando-se um espaçador de latão com o intuito de melhorar a uniformidade e regularidade na espessura do filme. Para reduzir ao máximo a degradação durante a preparação dos filmes foi utilizado o melhor par tempo-temperatura, sendo este de aproximada- mente 40 segundos de espera para fundir o polímero mais 10 segundos com uma pressão de 5 toneladas à temperatura de $190{ }^{\circ} \mathrm{C}$. Os filmes foram retirados da prensa e resfriados rapidamente em uma cuba de água com gelo para uniformizar a cristalização. As análises dos filmes foram feitos no modo de transmissão na faixa de 4000 a $400 \mathrm{~cm}^{-1}$ utilizando uma resolução de $4 \mathrm{~cm}^{-1}$. As soluções contendo produtos voláteis $\left(\mathrm{VOC}+\mathrm{CHCl}_{3}\right)$ foram analisadas por FTIR, via refletância total atenuada (ATR) com cristal de ZnSe, na faixa de 4000 a $620 \mathrm{~cm}^{-1}$ utilizando uma resolução de $4 \mathrm{~cm}^{-1}$. Antes da analise de cada amostra foi obtida o espectro de "linha base". $\mathrm{O}$ equipamento utilizado foi da marca Perkin-Elmer FTIR modelo Spectrum 1000.

\section{Resultados e Discussão}

\section{Curvas de distribuição de massa molar}

A Figura 2 mostra as curvas de distribuição de massa molar como uma função do volume de eluição das amostras do PP virgem e após extrusão nas diferentes temperaturas. O pico a aproximadamente $34,29 \pm 0,09 \mathrm{~mL}$ corresponde ao estabilizante térmico Irganox 1010, utilizado como marcador interno para analisar o erro causado pelo possível deslocamento entre as curvas, causado por mudanças na taxa de bombeamento. Encontrou-se uma variação máxima de 9\%, o que pode ser considerado desprezível frente aos deslocamentos entre as curvas de distribuição de massa molar gerados pela degradação termo-mecânica.

A seguir as curvas de volume de eluição foram convertidas em curvas de distribuição de massa molar (MWD) segundo a norma ASTM D 6474-99 e estão apresentadas na Figura 3. As curvas tiveram suas área normalizadas e delas foram calculadas as massas molares médias $\left(\overline{\mathrm{M}}_{\mathrm{n}}, \overline{\mathrm{M}}_{\mathrm{w}}, \overline{\mathrm{M}}_{\mathrm{z}}\right)$, polidispersão e a fração de cadeias clivadas, com os valores apresentados na Tabela 1. Analisando-se os resultados notase que o aumento da temperatura de extrusão provocou uma redução nas massas molares e que essa redução foi maior

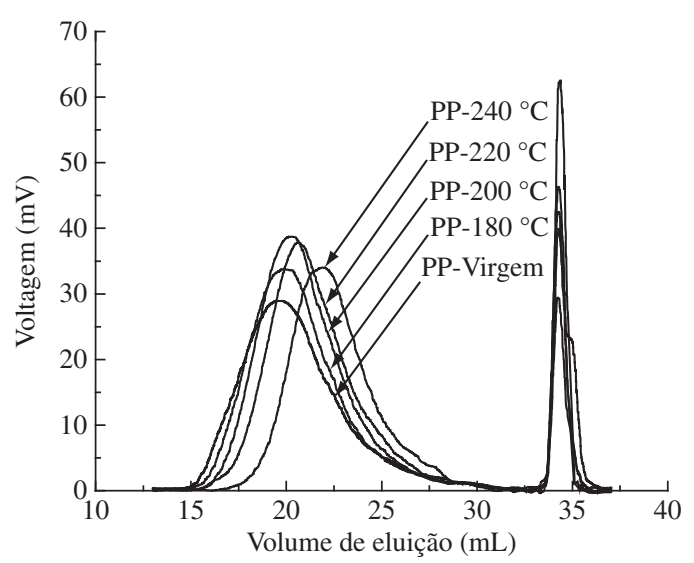

Figura 2. Curvas de volume de eluição (SEC) para o polipropileno virgem e após extrusão a diferentes temperaturas. O pico a aproximadamente $34,29 \pm 0,09 \mathrm{~mL}$ corresponde à eluição do marcador interno, indicando a alta estabilidade do fluxo. 


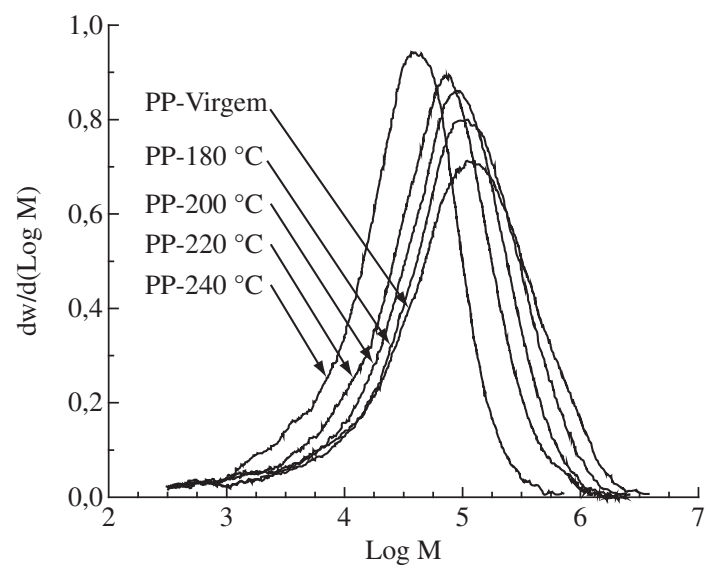

Figura 3. Curvas de distribuição de massas molar para PP virgem e extrudado em diferentes temperaturas, como indicado. Observar o deslocamento para a esquerda das curvas devido á degradação termo-mecânica.

nas médias de ordem superior, ou seja $\Delta \overline{\mathrm{M}}_{\mathrm{z}}>\Delta \overline{\mathrm{M}}_{\mathrm{w}}>\Delta \overline{\mathrm{M}}_{\mathrm{n}}$. A redução nas massas molares médias indica que a degradação termo-mecânica está causando preferencialmente a cisão das cadeias. A redução maior nas médias superiores indica que está havendo uma redução maior e preferencial na concentração das frações do polipropileno com mais alta massa molar, ou seja, a degradação termo-mecânica via cisão de cadeias é preferencial, ocorrendo com maior freqüência nas cadeias de maior massa molar. Este fato leva a uma redução na polidispersão que chega a $60 \%$ para o polipropileno processado na mais alta temperatura, i.e. $240{ }^{\circ} \mathrm{C}$. A fração de cadeias clivadas $f_{\mathrm{s}}$ é baixa nas temperaturas abaixo de $200{ }^{\circ} \mathrm{C}$, aumentando com o aumento da temperatura de extrusão. A $220^{\circ} \mathrm{C}$ um quarto das cadeias originais são clivadas e atingindo $75 \%$ delas a $240{ }^{\circ} \mathrm{C}$, ou seja, de cada quatro cadeias originais três sofreram uma cisão.

\section{Função de distribuição de cisão de cadeia}

A intensidade do deslocamento das curvas de MWD foi quantificada através da Função de Distribuição de Cisão de Cadeia (CSDF). Essa forma de apresentação dos dados calcula a redução da massa molar inicial do polipropileno (virgem) quando este é extrudado às diferentes temperaturas, como mostrado na Figura 4. As curvas de CSDF são afetadas pela temperatura de extrusão indicando que o processo de degradação termo-mecânico também o é. Amostras processadas a 180 e $200{ }^{\circ} \mathrm{C}$ apresentam baixos valores de CSDF, inclusive mostrando valores negativos no primeiro terço da escala de massa molar. Valores negativos de CSDF indicam

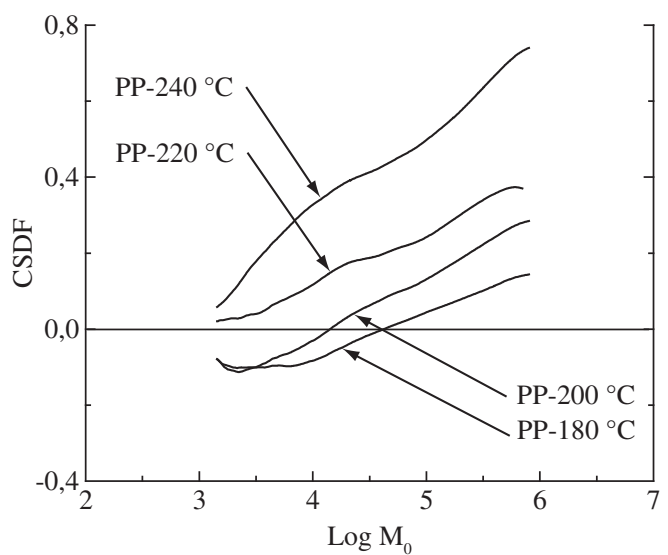

Figura 4. Curvas de CSDF do polipropileno extrudado a diferentes temperaturas, como indicado.

que a degradação apresenta um outro processo concorrente à cisão de cadeia, provavelmente um mecanismo de ramificação e/ou reticulação, similar ao processo de degradação termo-mecânico do $\mathrm{HDPE}^{[4]}$, mas de baixa intensidade. Este último comportamento pode ter sido favorecido pela presença de meros de etileno no copolímero propileno-etileno utilizado. Nos $2 / 3$ seguintes da escala de massa molar, apenas têm-se valores positivos de CSDF, o mesmo acontecendo para as amostras processadas a 220 e $240{ }^{\circ} \mathrm{C}$, indicando que o mecanismo de degradação termo-mecânica nesta faixa de massa molar e temperatura é predominantemente por cisão de cadeia. Por fim todas as curvas de CSDF são crescentes, ou seja, são dependentes da massa molar inicial, quanto maior o comprimento da cadeia maior a chance de formação de emaranhamentos e, por conseguinte maior é a chance das cadeias sofrerem cisão.

\section{Espectrometria no infravermelho}

Os produtos voláteis, gerados durante o processamento nas diferentes temperaturas, dissolvidos na forma de soluções concentradas em clorofórmio foram analisados no modo de refletância total atenuada (ATR). O peso de VOC, gerados durante as extrusões a 180 e $220{ }^{\circ} \mathrm{C}$, encontra-se na faixa de 25 a $50 \mathrm{mg}$ correspondendo a uma perda de massa de aproximadamente $0,01 \%$ para cada $0,5 \mathrm{~kg}$ de PP processado. A Figura 5 mostra os espectros de infravermelho do clorofórmio puro e das amostras dos voláteis coletados a 180, 200 e $220^{\circ} \mathrm{C}$. As faixas analisadas foram de 3000 a $2800 \mathrm{~cm}^{-1}$ e 1800 a $1600 \mathrm{~cm}^{-1}$ que correspondem a bandas de absorção de ligações $\mathrm{C}-\mathrm{H}$ e $\mathrm{C}=\mathrm{O}$ respectivamente. Os picos de

Tabela 1. Massas molares médias $\left(\overline{\mathrm{M}}_{\mathrm{n}}, \overline{\mathrm{M}}_{\mathrm{w}}, \overline{\mathrm{M}}_{\mathrm{z}}\right)$, polidispersão de primeira $\left(\overline{\mathrm{M}}_{\mathrm{w}} \overline{\mathrm{M}}_{\mathrm{n}}\right)$ e segunda $\left(\overline{\mathrm{M}}_{\mathrm{z}} / \overline{\mathrm{M}}_{\mathrm{w}}\right)$ ordem e fração $(f)$ de cadeias clivadas para polipropileno extrudado a diferentes temperaturas como indicado.

\begin{tabular}{ccrcccc}
\hline Polímero & $\overline{\mathbf{M}}_{\mathrm{n}}$ & $\overline{\mathbf{M}}_{\mathrm{w}}$ & $\overline{\mathbf{M}}_{\mathrm{z}}$ & $\overline{\mathbf{M}}_{\mathrm{w}} / \overline{\mathbf{M}}_{\mathrm{n}}$ & $\overline{\mathbf{M}}_{\mathbf{z}} / \overline{\mathbf{M}}_{\mathrm{w}}$ & $f_{\mathrm{s}}$ \\
\hline PP-Virgem & 28,000 & 216,000 & 551,000 & 7,70 & 2,60 & - \\
PP-180 ${ }^{\circ} \mathrm{C}$ & 30,700 & 171,000 & 395,000 & 5,60 & 2,30 & $-0,09$ \\
PP-200 ${ }^{\circ} \mathrm{C}$ & 27,800 & 128,000 & 277,000 & 4,60 & 2,20 & 0,01 \\
PP-220 ${ }^{\circ} \mathrm{C}$ & 22,300 & 92,500 & 301,000 & 4,20 & 3,30 & 0,25 \\
PP-240 ${ }^{\circ} \mathrm{C}$ & 16,000 & 52,200 & 96,400 & 3,30 & 1,90 & 0,75 \\
\hline
\end{tabular}



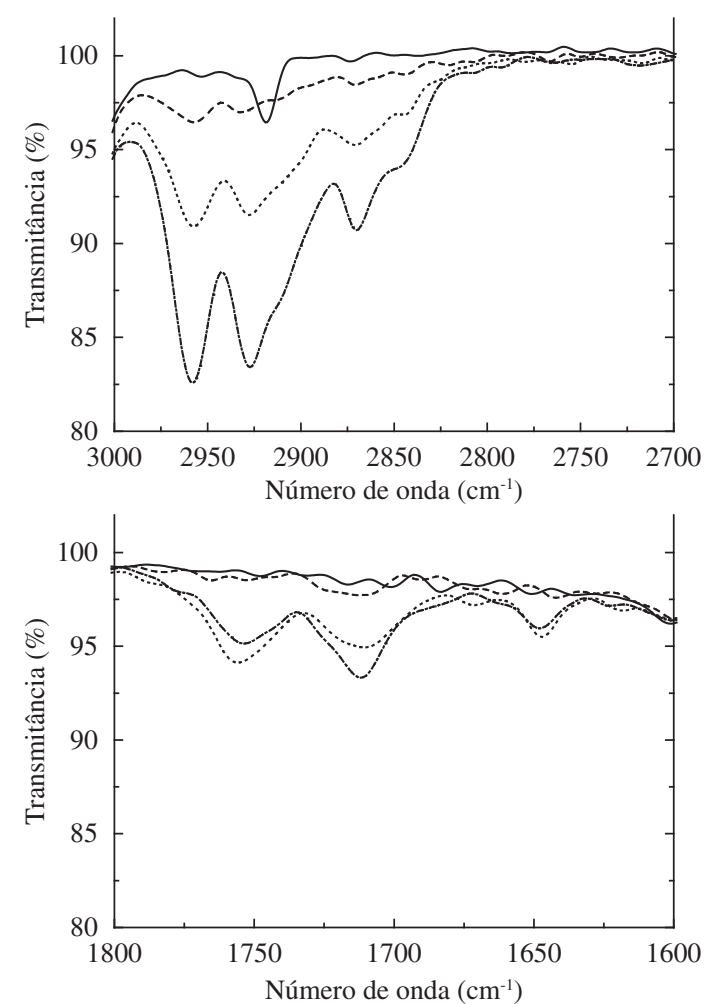

$$
\begin{array}{|lr|}
\hline-\mathrm{CHCl}_{3} & \text {-.-. } \mathrm{CHCl}_{3}+\text { Volat. }-180{ }^{\circ} \mathrm{C} \\
\text {........ } \mathrm{CHCl}_{3}+\text { Volat. }-200{ }^{\circ} \mathrm{C} & -\ldots-\mathrm{CHCl}_{3}+\text { Volat. }-220^{\circ} \mathrm{C} \\
\hline
\end{array}
$$

Figura 5. Espectros no infravermelho das soluções de voláteis em clorofórmio nas faixas de 3000 a $2800 \mathrm{~cm}^{-1}$ e 1800 a $1600 \mathrm{~cm}^{-1}$, correspondentes a ligações $\mathrm{C}-\mathrm{H}$ alifático e $\mathrm{C}=\mathrm{O}$, respectivamente.

transmitância característicos do $\mathrm{CHCl}_{3}$ não apresentam superposição com os picos dos voláteis gerados na extrusão do PP. No espectro da amostra processada a $180{ }^{\circ} \mathrm{C}$ se observa alguns picos de pouca intensidade correspondentes a estiramentos axiais e angulares das ligações $\mathrm{C}-\mathrm{H}$, não sendo ainda observadas bandas características de ligações $\mathrm{C}=\mathrm{O}$. Acima de $200{ }^{\circ} \mathrm{C}$ a concentração de compostos orgânicos voláteis aumenta, mostrando claramente picos a 2957 e $2871 \mathrm{~cm}^{-1}$ que corresponde à deformação axial assimétrica e simétrica do hidrogênio do grupo metila, respectivamente. Um pico a $2928 \mathrm{~cm}^{-1}$ também é observado e corresponde a deformação assimétrica do hidrogênio do grupo metileno. Picos a 1753, 1715 e $1647 \mathrm{~cm}^{-1}$ também são observados e correspondem a compostos carbonílicos e insaturações. Portanto, pode-se dizer que o polipropileno quando extrudado a temperaturas acima de $200{ }^{\circ} \mathrm{C}$ gera compostos voláteis olefínicos oxigenados, sendo que estes apresentam concentração crescente com o aumento da temperatura de processamento.

Amostras do polipropileno extrudado na forma de filmes foram analisadas no FTIR por transmissão. Assumindo-se que coeficientes de absorvitividade molar dos compostos analisados são os mesmos e para eliminar o efeito da espessura das amostras normalizou-se utilizando o pico de referência a $2720 \mathrm{~cm}^{-1}$, relacionado às vibrações angulares de $\mathrm{CH}$ e axial

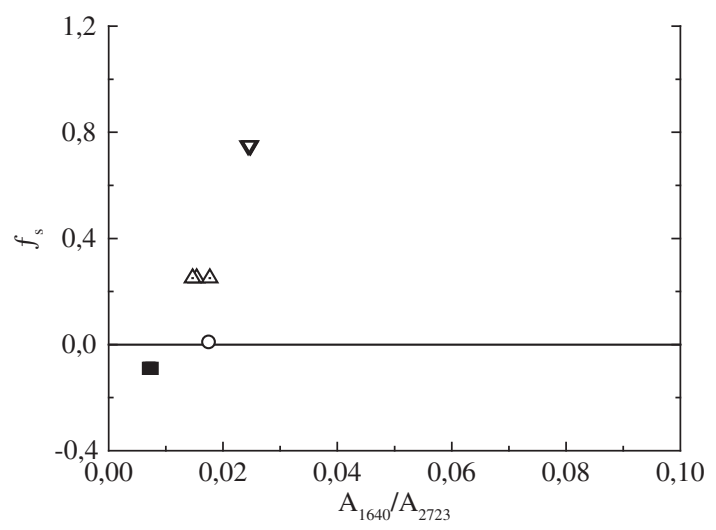

- $\mathrm{PP}-180^{\circ} \mathrm{C} \quad \circ \mathrm{PP}-200^{\circ} \mathrm{C} \quad \Delta \mathrm{PP}-220^{\circ} \mathrm{C} \quad \nabla \mathrm{PP}-240{ }^{\circ} \mathrm{C}$

(a)

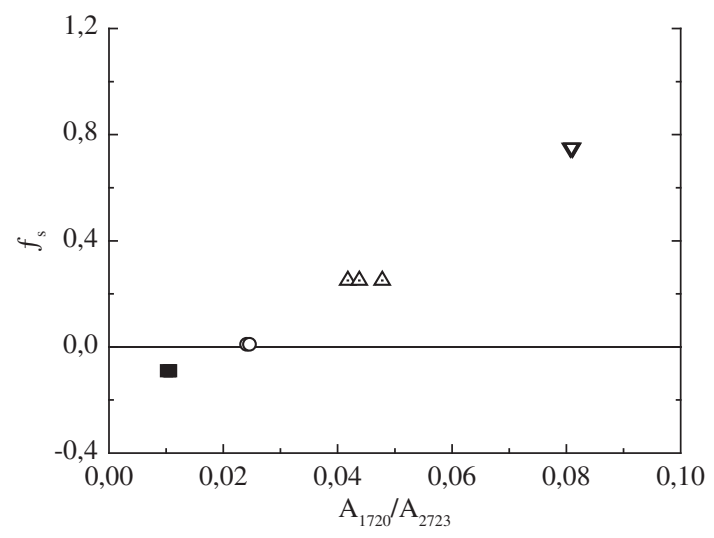

- $\mathrm{PP}-180^{\circ} \mathrm{C} \quad \circ \mathrm{PP}-200^{\circ} \mathrm{C} \quad \Delta \mathrm{PP}-220^{\circ} \mathrm{C} \quad \nabla \mathrm{PP}-240^{\circ} \mathrm{C}$

(b)

Figura 6. Correlação entre a fração de cadeias clivadas $f_{\mathrm{s}}$ e os: a) índices de insaturações; e b) carbonilas.

do grupo metila, insensíveis à cristalização e orientação ${ }^{[13-15]}$. A Figura 6 mostra a relação entre a fração de cadeias clivadas $f_{\mathrm{s}}$ e a absorção normalizada de insaturações e carbonilas segundo $\mathrm{A}_{1640} / \mathrm{A}_{2720}$ e $\mathrm{A}_{1720} / \mathrm{A}_{2720}$, conhecidos como Índice de Insaturações (Iins) e Índice de Carbonilas (Ic), respectivamente. $\mathrm{O}$ índice de carbonilas aumenta com a temperatura de processamento e apresenta uma variação linear com o número de cisões de cadeia. $O$ índice de insaturações segue o mesmo comportamento, mas com menor intensidade. Um aumento da temperatura para 220 e $240{ }^{\circ} \mathrm{C}$ ocasiona um aumento expressivo no índice de carbonilas, o que não ocorre com o índice de insaturações que apresenta um leve aumento. Isto indica que a degradação termo-mecânica do polipropileno durante a extrusão leva com maior freqüência à oxidação das cadeias que à formação de insaturações.

\section{Conclusões}

O polipropileno copolímero aleatório de propeno - eteno, sem a adição de estabilizantes térmicos, quando extrudado sofre degradação termo-mecânica, reduzindo as massas mo- 
lares médias com o aumento da temperatura de processamento. A fração das cadeias clivadas só se torna significativa a temperaturas de extrusão acima de $220{ }^{\circ} \mathrm{C}$. A Função de Distribuição de Cisão de Cadeia sugere que às temperaturas de extrusão abaixo de $220{ }^{\circ} \mathrm{C}$ o mecanismo de cisão de cadeia não parece ser único indicando a existência de um mecanismo de ramificação e/ou reticulação. No entanto, a partir de $220{ }^{\circ} \mathrm{C} \mathrm{o}$ mecanismo de cisão de cadeias torna-se predominante e as curvas de CSDF indicam que o processo de degradação termo-mecânico é dependente da massa molar inicial e da temperatura de extrusão, ou seja, um processo de degradação do tipo preferencial. A degradação também gera compostos orgânicos voláteis VOC, detectados para temperaturas de extrusão acima de $200{ }^{\circ} \mathrm{C}$. Os VOC coletados correspondem a compostos olefínicos com massas molares não voláteis à temperatura ambiente, sendo principalmente compostos oxigenados como carbonilas e insaturações. Com o aumento da temperatura de extrusão incrementou-se a concentração dos VOC coletados. O peso dos VOC coletados se encontra na faixa de 25 a $50 \mathrm{mg}$ para cada $0,5 \mathrm{~kg}$ de polipropileno processado. À temperatura de $220{ }^{\circ} \mathrm{C}$ a perda de material por volatilização foi de $0,01 \%$ (w/w).

\section{Agradecimentos}

Agradecemos ao CNPq/FINEP pelo apoio financeiro, ao $\mathrm{CNPq}$ pela bolsa de estudos (C. A. C. C) e à Braskem S.A.pela doação do polipropileno copolímero.

\section{Referências Bibliográficas}

1. Moore, E. P. - "Polypropylene Handbook", Hanser Publishers, New York (1996).

2. Hinsken, H.; Moss, S.; Pauquet J. R. \& Zweifel, H. Polym. Degrad. Stab., 34, p.279 (1991).
3. Pinheiro, L. A.; Chinelatto, M. A. \& Canevarolo, S. V. Polym. Degrad. Stab., 86, p.445 (2004).

4. Xiang, Q.; Xanthos, M.; Mitra, S.; Patel, S. H. \& Guo, J. - Polym. Degrad. Stab., 77, p.93 (2002).

5. Barlow, A.; Contos, D.; Holdren, M. W.; Garrison, P. J.; Harris, L. R. \& Janke, B. - J. Air \& Waste Manage. Assoc., 46, p.569 (1996).

6. Barlow, A.; Moss, P.; Parker, E.; Schoroer, T.; Holdren, M. \& Adams, K. - J. Air \& Waste Manage. Assoc., 47, p.1111 (1997).

7. Adams, K.; Bankston, J.; Barlow, A.; Holdren, M. W.; Meyer, J. \& Marchesani, V. J. - J. Air \& Waste Manage. Assoc., 49, p.49 (1999).

8. Cáceres, C. A. \& Canevarolo, S. V. - Polímero: Ciência \& Tecnologia, Aceito para publicação (2008).

9. Canevarolo, S. V. - Polym. Degrad. Stab., 709, p.71 (2000).

10. Cáceres, C. A. \& Canevarolo, S. V. - Polym. Degrad. Stab., 86, p.437 (2004).

11. Cáceres, C. A. \& Canevarolo, S. V. - Polímero: Ciência \& Tecnologia, 16, p.294 (2006).

12. Machado, A.; Maia, J.; Canevarolo, S. V. \& Covas, J. Apply. Polym. Sci., 91, p.2711 (2004).

13. Rabello, M. S. \& White, J. R. - Polym. Degrad. Stab., 56, p.55 (1997).

14. Garton, A.; Carlsson, D. J. \& Wiles, D. M. - J. Polym. Sci. Polym. Chem., 16, p.33 (1978).

15. Tobin, M. C. - J. Phys. Chem., 64, p.216 (1960).

Enviado: $26 / 12 / 07$

Reenviado: 01/10/08

Aceito: $24 / 11 / 08$ 